\title{
Evaluation of the hemocompatibility of hydrated biodegradable aliphatic carbonyl polymers with a subtle difference in the backbone structure based on the intermediate water concept and surface hydration
}

\author{
Polymer Journal (2015) 47, 469-473; doi:10.1038/pj.2015.22; published online 15 April 2015
}

\section{INTRODUCTION}

Synthetic biodegradable polymers have been broadly studied in recent decades for application in biomedical devices such as orthopedic treatments, ${ }^{1}$ drug delivery ${ }^{2}$ and tissue engineering. ${ }^{3}$ Commonly used biodegradable polymers such as poly(lactic acid), $\operatorname{poly}(\varepsilon-$ caprolactone) (PCL), poly(dioxanone) (PDO) and poly(trimethylene carbonate) (PTMC) comprise ester or carbonate linkages and alkyl or alkyloxy chains, which enable facile degradation in aqueous environments with and without enzymes. ${ }^{4}$

A crucial feature of medical devices is their compatibility with the host cells to reduce adverse effects; the aforementioned polymers exhibit minimal or acceptable cytotoxicity. ${ }^{5}$ However, few reports have described the relationship between the biocompatibility, particularly hemocompatibility, and the structural features of these polymers. In recent years, a PTMC analog with an ammonium side group was reported to have potent antimicrobial activities with minimal hemolytic properties. ${ }^{6}$ By contrast, (meth)acrylatebased cationic polymers often exhibit some degree of hemolytic activity. ${ }^{7}$ Thus, we deduced that the backbone structure of these aliphatic carbonyl polymers has a potential role in reducing the cytotoxicity that cationic materials usually exhibit.

Recently, surface hydration layers have been recognized as a critical factor in mitigating biological responses to biomaterials. We have previously reported that intermediate water', which is defined as water that is weakly bound to a polymer in the hydration layer of poly(2-methoxyethyl acrylate) (PMEA), is responsible for its excellent hemocompatibility, as evidenced by the poor adhesion of platelets and fibrinogen to this material. ${ }^{8}$ The intermediate water is observed in other biocompatible polymers such as biopolymers ${ }^{9}$ and readily analyzed using differential scanning calorimetry (DSC) of hydrated polymers. Although DSC does not provide accurate quantitative information about the actual surface hydration, a correlation was found between the degree of platelet adhesion and the amount of intermediate water, which was determined using DSC. ${ }^{10}$

Polymer hydration occurs through hydrogen bonding between polar moieties in the polymer and water molecules. For PMEA, the ester groups on the side chains primarily contribute to generating nonfreezing water, which is firmly bound to a polymer, because of a bridging hydrogen bonding with two side chains $(\mathrm{C}=\mathrm{O} \bullet \bullet \cdot \mathrm{H}-\mathrm{O}-\mathrm{H} \bullet \bullet \cdot \mathrm{O}=\mathrm{C}) .{ }^{10}$ However, the ester groups in the main chain do not behave in identical manners $\left(\mathrm{C}=\mathrm{O} \bullet \bullet \mathrm{H}_{-}\right.$ $\mathrm{O}-\mathrm{H})$, and we assume that the water that interacts with water molecules that are strongly bound to $\mathrm{C}=\mathrm{O}$, which is called secondary bound water $(\mathrm{C}=\mathrm{O} \bullet \bullet \bullet-\mathrm{H}-(\mathrm{HO})$ $\cdots \mathrm{H}-\mathrm{O}-\mathrm{H}$ ), behaves like intermediate water. In this report, we study the hydration behavior, intermediate water content and platelet adhesion level of PTMC and three other aliphatic carbonyl polymers (PDO, PCL and $\operatorname{poly}(\delta$-valerolactone) $(\mathrm{PVL}))$ to elucidate the influence of subtle differences in the backbone structure on hydration and hemocompatibility.

\section{MATERIALS AND METHODS}

PCL $\left(M_{\mathrm{n}} 70-100 \mathrm{k}\right)$ and PDO (molecular weight information is not provided) were commercially obtained from Wako Pure Chemical Industries (Osaka, Japan) and Sigma-Aldrich (St Louis, MO, USA), respectively, and used as received. PTMC $\left(M_{\mathrm{n}} 22000\right)$ and PVL $\left(M_{\mathrm{n}} 28500\right)$ were synthesized as reported elsewhere. ${ }^{11}$ The reprecipitated and triturated polymers were used as DSC specimens. A spin-coated surface was used for the platelet adhesion test and contact angle measurements. The cast coatings and films were used for the infrared spectroscopy (IR). All hydrated surfaces and films were prepared by immersion in deionized water for $24 \mathrm{~h}$.

The phase transitions of water in the hydrated polymers were measured using DSC. The overall water content in the polymer was determined as follows: $W_{\mathrm{p}}=\left(\left(w_{1}-w_{0}\right) / w_{1}\right) \times 100 \quad(\mathrm{wt} \%)$, where $w_{1}$ and $w_{0}$ are the weights of a hydrated sample and a dry sample, respectively. Each water content was derived from $W_{\mathrm{p}}$, which is the observed heat of crystallization of water $\left(\Delta H_{\mathrm{c}}\right)$, observed heat of melting of ice $\left(\Delta H_{\mathrm{m}}\right)$ and heat of fusion of perfect ice $\left(334 \mathrm{Jg}^{-1}\right)$ as described elsewhere, ${ }^{9}$ using the relationship: $W_{\mathrm{p}}=W_{\mathrm{nf}}+W_{\mathrm{im}}+W_{\mathrm{f}}=W_{\mathrm{nf}}+\Delta H_{\mathrm{c}} / 344+$ $\left(\Delta H_{\mathrm{m}}-\Delta H_{\mathrm{c}}\right) / 344 . W_{\mathrm{im}}, W_{\mathrm{f}}$ and $W_{\mathrm{nf}}$ denote the contents of intermediate, free and nonfreezing waters, respectively.

The roughness of the spin-coated surfaces was evaluated using atomic force microscopy. The static contact angles of the polymer surface against water were measured using both a sessile drop and a captive bubble. Fourier transform infrared (FT-IR) spectra were recorded in the transmission mode for the polymer, which was casted on glass substrates at the dry and hydrated state, and a blank glass substrate was used as a reference.

\section{RESULTS AND DISCUSSION}

First, we investigated the thermal histories of water in the hydrated polymers (Figure 1a). The typical DSC heating and cooling scans of the polymers at equilibrium water content (EWC) are presented in Figures $1 \mathrm{~b}$ and $\mathrm{c}$, 
a<smiles>CC(C)CCCCCC(C)(C)C</smiles>

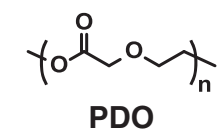

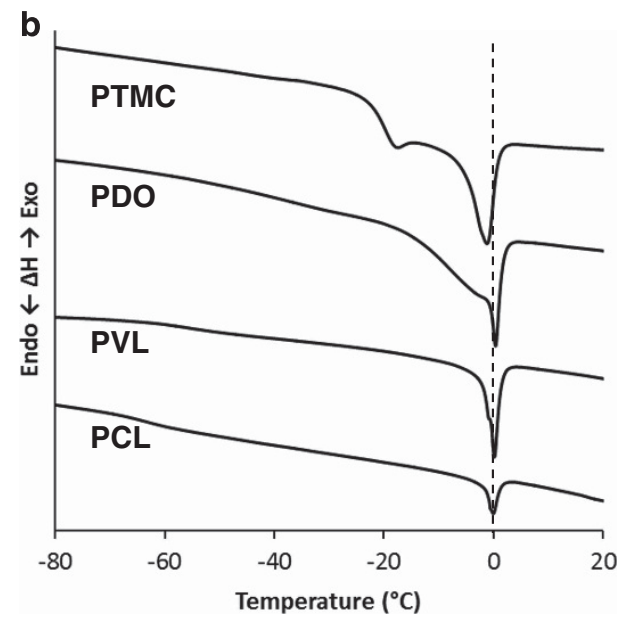

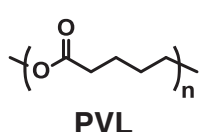<smiles>CC(=O)OCCCCC(C)(C)C</smiles>

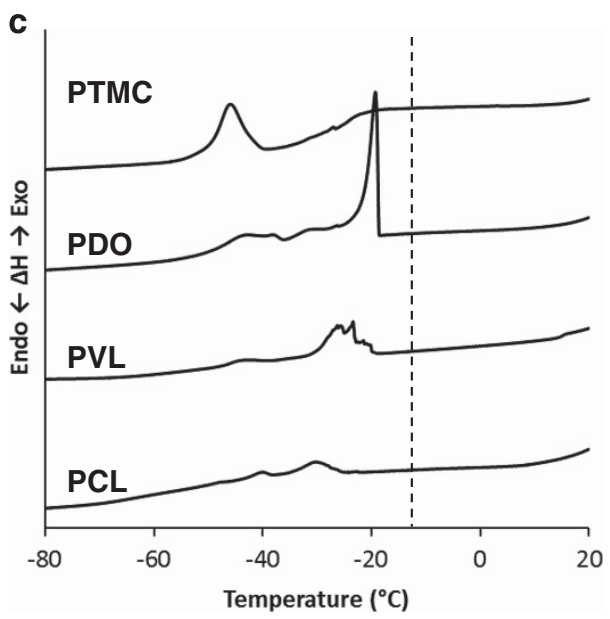

Figure 1 Structure (a) and typical differential scanning calorimetry curves $(\mathbf{b}, \mathbf{c})$ of the hydrated aliphatic carbonyl polymers at equilibrium water content: PTMC, PDO, PVL and PCL. Heating scans (b) and cooling scans (c), which were run with a ramp of $5{ }^{\circ} \mathrm{C} \mathrm{min}^{-1}$. The dotted lines depict the temperatures for the melting of ice and freezing of water. PCL, poly( $\varepsilon$-caprolactone); PDO, poly(dioxanone); PTMC, poly(trimethylene carbonate); PVL, poly( $\delta$-valerolactone).

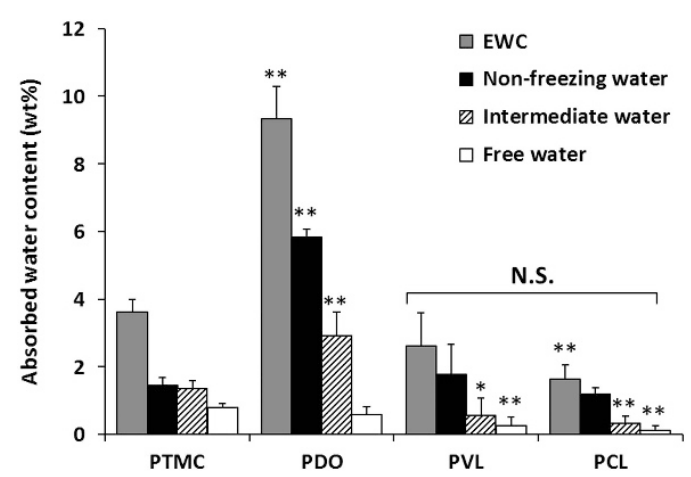

Figure 2 Absorbed water contents of each water type at EWC in the hydrated aliphatic carbonyl polymers (mean \pm s.d.; $n=3$ ). ${ }^{*} P<0.01$ and ${ }^{*} P<0.05$ vs PTMC. EWC, equilibrium water content; NS, not significant; PTMC, poly(trimethylene carbonate).

respectively. Although none of the polymers showed exothermic crystallization, some degree of endothermic melting below $0{ }^{\circ} \mathrm{C}$ was found in the heating scans (Figure 1b), which implies that the formation of intermediate water is induced by the interaction with the polymer structure. During the cooling step (Figure 1c), distinct exothermic peaks were observed from -20 to $-50{ }^{\circ} \mathrm{C}$, which are clearly lower than the crystallization temperature of bulk water $\left(-12^{\circ} \mathrm{C}\right.$, Supplementary Figure S1). These crystallization peaks also appear to be well associated with the endotherms below $0{ }^{\circ} \mathrm{C}$ in the heating scans. Therefore, we used the exothermic peaks in the cooling scans to calculate the amount of intermediate water. A similar behavior has been observed for other hydrated polymers. ${ }^{12}$ PTMC showed a distinct single crystallization peak on the heating scan (Figure 1b). By contrast, the other polymers showed broad endotherms, which implies a singularity of carbonate bonds on interacting with water.

Figure 2 shows the amount of absorbed water in each hydrated polymer. The EWC and water contents for each sample were determined through at least five measurements of fully hydrated samples $(n=3)$. PDO was highly hydrated compared with the other polymers and showed the highest EWC likely attributable to the ether bonds in the main chain. ${ }^{13}$ The high intermediate water content of PDO is consistent with its clinical use in biodegradable stents, which exhibit hemocompatibility. ${ }^{5}$ By contrast, PTMC showed a much lower EWC than PDO $(9.3 \mathrm{wt} \%)$. The hydration of PDO is most
(3.6 wt\%) despite the identical atomic compositions. This result also suggests a strong contribution of ether bonds to hydration. PVL showed a lower EWC (2.6 wt\%), probably because of the lack of one oxygen. Although PTMC and PVL had similar EWCs and nonfreezing water contents, the intermediate water contents were significantly different $(1.4 \mathrm{wt} \%$ for PTMC vs $0.6 \mathrm{wt} \%$ for PVL), which indicates that the carbonate bond is more capable of forming intermediate water. PCL contains the lowest amount of intermediate water, presumably because of its high hydrophobicity.

Next, we evaluated the platelet adhesion capacity of the polymer surfaces. In addition to PMEA, poly(ethylene terephthalate) and poly(2-methacryloyloxyethyl phosphorylcholine-co-butyl methacrylate) were used as control polymers. The thickness of the spin coating is controllable with a certain concentration of polymer solution regardless of the polymer's characteristics, for example, a thickness of $160 \mathrm{~nm}$ for $0.5 \mathrm{wt} \%$. Platelets adhered on all four aliphatic carbonyl polymers and the adherent platelets were activated with pseudopodia (Supplementary Figure S2), as found on the poly(ethylene terephthalate) surfaces (Supplementary Figure S3). Although we confirmed a certain level of smoothness for each polymer surface using scanning electron microscopy (Supplementary Figure S4) and measured the roughness using atomic force microscopy (Table 1), which showed that there were minimal differences among 
Table 1 Surface and bulk characteristics of the aliphatic carbonyl polymers

\begin{tabular}{|c|c|c|c|c|c|c|c|c|}
\hline & \multicolumn{4}{|c|}{ Spin-coated surface } & \multicolumn{4}{|c|}{ Bulk samples } \\
\hline & \multirow[b]{2}{*}{$R M S(n m ; \pm s . d .)^{a}$} & \multicolumn{3}{|c|}{ Contact angle (degrees; \pm s.d. $)^{b}$} & \multicolumn{2}{|c|}{$\mathrm{T}_{g}\left({ }^{\circ} \mathrm{C}\right)$} & \multicolumn{2}{|c|}{$\mathrm{T}_{m}\left({ }^{\circ} \mathrm{C}\right)$} \\
\hline & & Sessile drop $(\theta)$ & Captive bubble ( $\phi)$ & $\theta+\phi$ & Dry & Hydrated & Dry & Hydrated \\
\hline PTMC & $73.0( \pm 5.0)$ & $72.9( \pm 1.1)$ & $121.5( \pm 1.1)$ & 194.5 & -17 & -20 & NA & NA \\
\hline PDO & $60.2( \pm 1.7)$ & $61.1( \pm 0.4)$ & $119.5( \pm 0.8)$ & 180.6 & -13 & -37 & 113 & 91 \\
\hline
\end{tabular}

Abbreviations: NA, not applicable; PCL, poly(e-caprolactone); PDO, poly(dioxanone); PTMC, poly(trimethylene carbonate); PVL, poly( $\delta$-valerolactone); RMS, root mean squared roughness. $a_{n=3}$. ${ }^{b} n=5$.
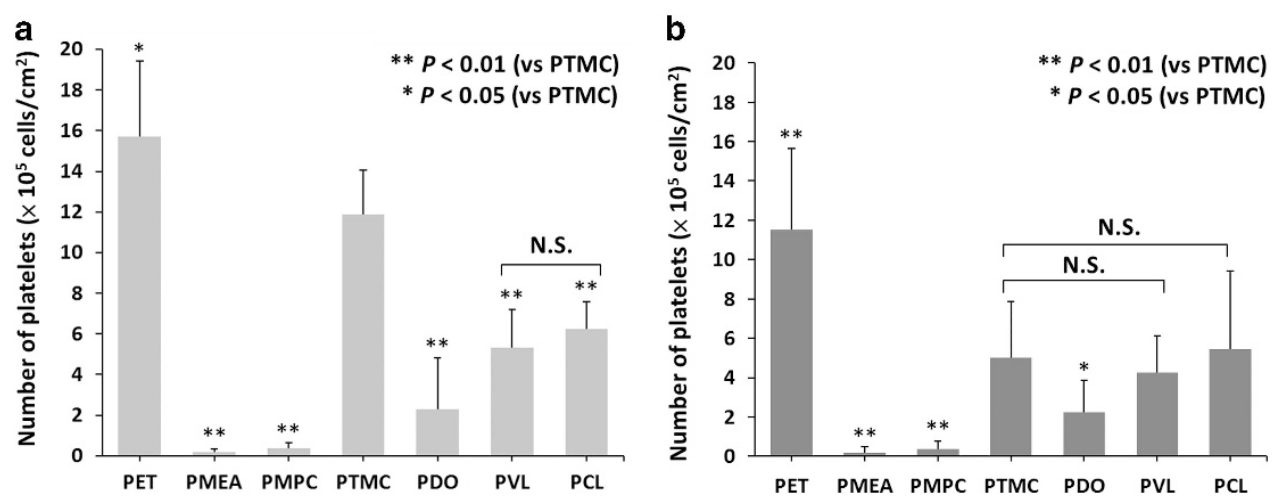

Figure 3 Numbers of platelets adhered to the polymer surfaces in the dry state (a) and hydrated state (b), which were primed with deionized water for $24 \mathrm{~h}$; mean \pm s.d. $(n=5) .{ }^{*} P<0.01$ and ${ }^{*} P<0.05$ vs PTMC. NS, not significant; PTMC, poly(trimethylene carbonate).

the four polymers, bumps were formed on the PTMC surfaces and some PMEA surfaces after the test (Supplementary Figures S2A and $\mathrm{S} 3 \mathrm{D})$. However, we concluded that these bumps negligibly affected the platelet adhesion because PMEA showed low platelet adhesion regardless of the existence of the bumps.

Figure $3 \mathrm{a}$ displays the number of adherent platelets on the dry polymer surfaces. Although the numbers were high, all aliphatic carbonyl polymers showed lower platelet adhesion than poly(ethylene terephthalate). Among the four polymers tested, PDO adsorbed the fewest platelets, as expected from its highest intermediate water content. By contrast, PTMC adsorbed significantly more platelets than any other polymer except poly(ethylene terephthalate). This result is in contrast to the theoretical order for platelet adhesion based on the intermediate water content: PCL $\sim$ PVL $>$ PTMC $>$ PDO. Therefore, intermediate water is not as segregated on the PTMC surface as estimated from DSC, which is different from the case of PMEA. ${ }^{14}$

To obtain a more rational explanation for the platelet adhesion results, we analyzed the contact angles against water for the polymer surfaces (Table 1). The initial state of the surface dominates the initial adsorption of plasma proteins and platelets. Furthermore, it has recently been found that the surface property of non-ionic polymers such as PMEA with plasma can be approximated by that in water. ${ }^{15}$ Thus, the sessile drop method and captive bubble method were used with deionized water to evaluate the dry surfaces and hydrated surfaces immersed in deionized water for $24 \mathrm{~h}$, respectively. The order of hydrophilicity for the dry surfaces was $\mathrm{PDO}>\mathrm{PVL}>\mathrm{PCL}>\mathrm{PTMC}$, which better correlates with the platelet adhesion results (Supplementary Figure S5A). The contact angles for the hydrated surfaces presented a different trend, whereby PTMC became more hydrophilic than PDO. The sum of only contact angles for PTMC far exceeded $180^{\circ}$ (Table 1), which suggests that the local conformation on the PTMC surface dramatically changes by hydration.

Then, we examined the platelet adhesion on the hydrated surfaces; the results are shown in Figure 3b. Overall, the platelet adherence slightly decreased $(\sim 25 \%)$ for all hydrated surfaces, and a remarkable decrease (58\%) was observed for the PTMC surface. However, the order of platelet adhesion $(\mathrm{PDO}<\mathrm{PVL} \sim$
PCL $\sim$ PTMC) remained inconsistent with the corresponding contact angles when a captive bubble is used. By contrast, the platelet adhesion behavior on the hydrated surfaces showed better correlations with both the surface wettability of the dry surface and the water contents (Supplementary Figures S5B and F), probably because the water contents were determined on fully hydrated polymer samples using DSC. The adsorption and denaturation of fibrinogen or plasma proteins on the hydrated surfaces were preliminarily examined using bicinchoninic acid assays and an enzyme-linked immunosorbent assay. PTMC, PVL and PCL showed a similar tendency to the platelet adhesion on the hydrated surfaces (Supplementary Figure S6). However, because of the difficulty in containing the crystallization for drop casting in a 96-well plate, some rough surfaces were formed, which resulted in the inconsistency with platelet adhesion particularly for PDO. Further improvement will be explored in future studies.

The decreased platelet adhesion for the hydrated PTMC is fascinating and can be induced specifically by the carbonate bond. The comparatively low molecular weight of PTMC may affect the platelet adhesion. However, such a significant decrease in the 

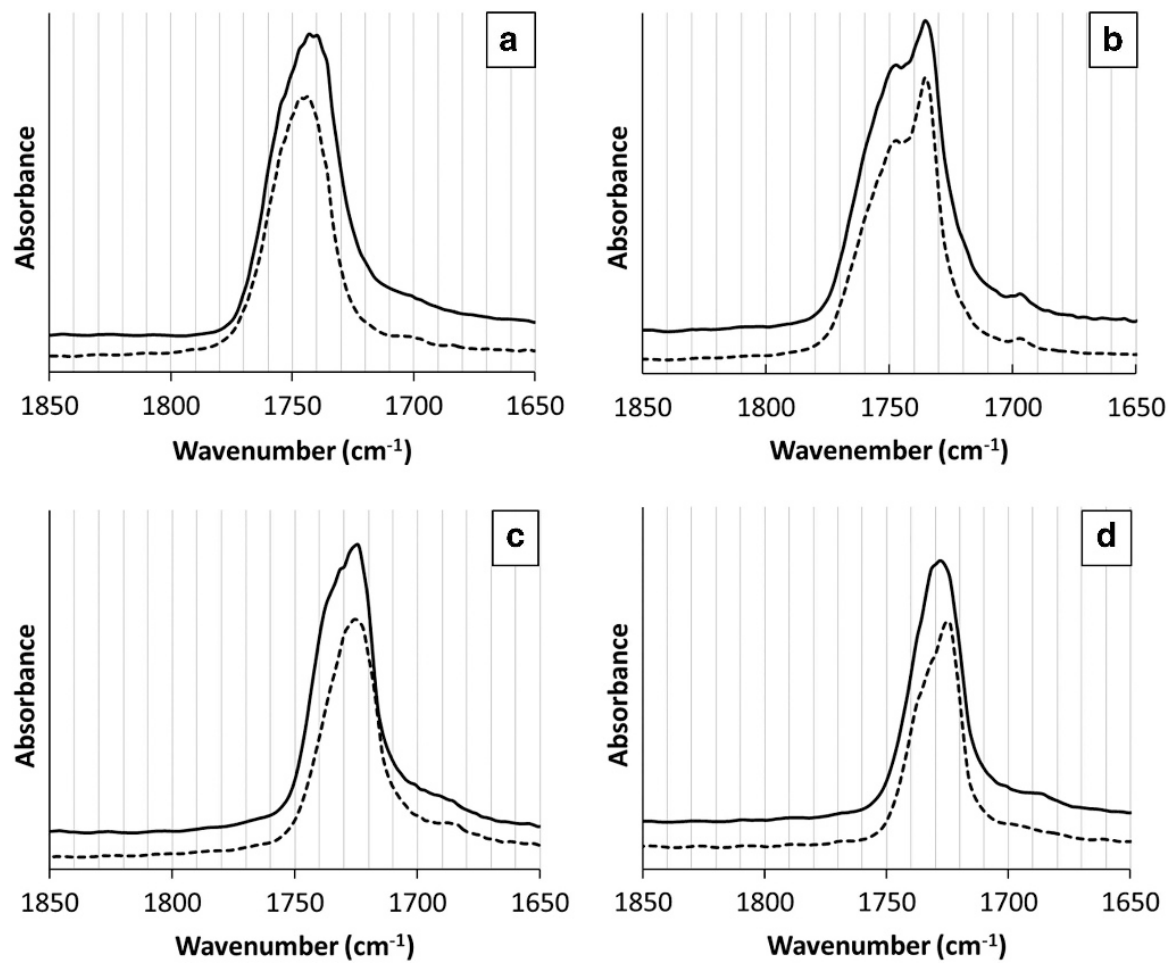

Figure 4 Expanded region of infrared spectra for the $\mathrm{C}=0$ stretching bands in PTMC (a), PDO (b), PVL (c) and PCL (d) in the dry state (dotted lines) and after priming in deionized water for $24 \mathrm{~h}$ (solid lines). PCL, poly(e-caprolactone); PDO, poly(dioxanone); PTMC, poly(trimethylene carbonate); PVL, poly ( $\delta$-valerolactone).

number of adhered platelets was not observed for PVL, which has a similarly low molecular weight (Figure 3b). To further understand the specificity of carbonate bonds, we investigated the hydration of the polymer cast coatings on a thin glass using FT-IR in the transmission mode. Each coating thickness was adjusted to $\sim 10 \mu \mathrm{m}$ (Supplementary Table S1). It has been reported that the characteristic $\mathrm{C}=\mathrm{O}$ stretching band shifts to a lower frequency upon hydrogen bonding. ${ }^{14}$ As shown in Figure 4, only PTMC showed a distinct lowfrequency shift of $5 \mathrm{~cm}^{-1}$ of the $\mathrm{C}=\mathrm{O}$ stretching band upon hydration, whereas the other aliphatic carbonyl polymers showed no shifts to lower frequency. For PDO, which is plasticized upon hydration with considerable decreases in $T_{\mathrm{g}}$ and $T_{\mathrm{m}}$ (Table 1), the ether bond must be predominantly involved in the hydration and the formation of intermediate water. However, the shift was not observed in the attenuated reflection infrared (ATR-IR) spectra (Supplementary Figure S7) for all hydrated polymer cast films with thickness of $\sim 100 \mu \mathrm{m}$ (Supplementary Table S1), which indicates that the conformational change on the surface is notably little compared with the change inside, and the ordinary ATR-IR technique could not observe the actual water-polymer interface.
In situ measurement under a high-humidity condition $^{10,14}$ is necessary to monitor the surface hydration in detail and will be reported in the future. Nonetheless, the FT-IR results support the notion that carbonate bonds are specifically liable to conformational change by hydration, which is in contrast to ester bonds, and the IR shift is likely associated with the decreased platelet adhesion and the sum of contact angles over $180^{\circ}$ for PTMC. Therefore, we presumed that a certain conformational change occurs at the water-PTMC interface.

One plausible interpretation of the specificity of PTMC on hydration is speculated from the recent finding at the water-polymer interface of poly(methyl methacrylate) (PMMA). The less-regulated $\mathrm{C}=\mathrm{O}$ in syndiotactic dominant PMMA (st-PMMA), which forms relaxed $37 / 4$ helices, ${ }^{16}$ is more susceptible to the approach of water, whereas the ordered $\mathrm{C}=\mathrm{O}$ in isotactic PMMA (it-PMMA), which forms 10/1 helices, is not subjected to conformational change by the approaching water molecules. ${ }^{17}$ All three aliphatic polyesters used in this study are semicrystalline, which form ordered structures of folding chains with a zigzag configuration and oriented ester groups. ${ }^{18,19}$ Presumably, such ester bonds in the ordered structure are stable and not disordered by water adsorption. By contrast, PTMC is amorphous and $\mathrm{C}=\mathrm{O}$ should be rarely oriented on the surface; thereby, the conformational change readily occurs with the adsorbed water molecules. The $\mathrm{C}=\mathrm{O}$ orientation also affects the dipole moment on the surface, which can explain the hydrophilicity of the PTMC surface and its change from the dry state to the hydrated state.

In addition, the extra oxygen may make the $\mathrm{C}=\mathrm{O}$ of the carbonate bonds more capable of acting as hydrogen-bonding acceptors than ester bonds. The higher hydrogen-bonding capability of PTMC also likely affects the formation of intermediate water, particularly the aforementioned secondary bound water. The shape and position of the peaks on the DSC charts provide information about the type and stability of the water that is bound to the polymer. PTMC shows a single distinct exotherm, whereas the other polyesters exhibit several broad exotherms in the cooling scans (Figure 1b), which suggests that PTMC has specific sites for strongly binding water, whereas the other polyesters have multiple interaction sites including hydrophobic hydration. Moreover, the exotherm at lower temperatures represents the potent hydrogen bonding between PTMC and water.

Nonetheless, PTMC demonstrates a similar degree of platelet adhesion to PVL and PCL even on the hydrated surface. Then, we must deliberate the inconsistency between the intermediate water content and platelet adhesion. Considering all foregoing results, PTMC possibly forms a quasi-fluid layer that comprises polymer chains and water at the interface, which is similar to PMEA and poly (2-methacryloyloxyethyl phosphorylcholineco-butyl methacrylate). According to a calculation based on the experimental results, for PMEA, the intermediate water is localized in the superficial layer in the formed quasi-fluid layer. ${ }^{14,20}$ However, for PTMC, the intermediate water probably spreads over the quasi-fluid layer, which leads to the error in the estimation for interfacial hydration using DSC. Consequently, the actual amount of intermediate water on the PTMC surface is lower than the DSC-calculated amount, which results in the similar platelet adhesion properties to that of PVL and PCL for the hydrated surfaces.

\section{CONCLUSION}

The effect of subtle differences in the backbone structures of PTMC, PDO, PVL and PCL on hydration was investigated. The ether 
bonds in the main chain of PDO are strongly related to hydration because of the increased generation of intermediate water. Moreover, by investigating the differences in hydration behavior of the polymer backbones with ester and carbonate bonds, we found that the carbonate bonds favor conformational change compared with ester bonds because of their stronger capacities as hydrogen bond acceptors and the less-regulated $\mathrm{C}=\mathrm{O}$ on the surface based on its amorphous nature. We believe that this study will help elucidate the roles of intermediate water in determining blood compatibilities and contribute to the sophisticated design of synthetic biodegradable polymers with excellent hemocompatibility.

\section{ACKNOWLEDGEMENTS}

This study was financially supported by a JSPS Grant-in-Aid for Young Scientists (B) \#25870078. MYT thanks the National Taiwan University for the travel grants. This work was also supported by the Funding Program for the Next Generation World-Leading Researchers (NEXT Program) from the Ministry of Education, Culture, Sports, Science and Technology (MEXT), Japan.

Kazuki Fukushima1, Meng-Yu Tsai ${ }^{2}$, Takayuki Ota ${ }^{2}$, Yuta Haga ${ }^{1}$, Kodai Matsuzaki ${ }^{1}$, Yuto Inoue ${ }^{2}$ and Masaru Tanaka ${ }^{1,2}$

${ }^{1}$ Department of Polymer Science and Engineering, Graduate School of Science and Engineering, Yamagata University, Yonezawa,
Yamagata, Japan and ${ }^{2}$ Department of Biochemical Engineering, Graduate School of Science and Engineering, Yamagata University, Yonezawa, Yamagata, Japan E-mail: fukushima@yz.yamagata-u.ac.jp or tanaka@yz.yamagata-u.ac.jp

1 Middleton, J. C. \& Tipton, A. J. Synthetic biodegradable polymers as orthopedic devices. Biomaterials 21, 2335-2346 (2000).

2 Soppimath, K. S., Aminabhavi, T. M., Kulkarni, A. R. \& Rudzinski, W. E. Biodegradable polymeric nanoparticles as drug delivery devices. J. Control. Release 70 , 1-20 (2001).

3 Hubbell, J. A. Synthetic biodegradable polymers for tissue engineering and drug delivery. Curr. Opin. Solid State Mater. Sci. 3, 246-251 (1998).

4 Naira, L. S. \& Laurencin, C. T. Biodegradable polymers as biomaterials. Prog. Polym. Sci. 32, 762-798 (2007).

5 Tzoneva, R., Seifert, B., Behl, M. \& Lendlein, A. Elastic multiblock copolymers for vascular regeneration: protein adsorption and hemocompatibility. Clin. Hemorheol. Micro. 52, 337-348 (2012).

6 Nederberg, F., Zhang, Y., Tan, J. P. K., Xu, K., Wang, H., Yang, C., Gao, S., Guo, X. D., Fukushima, K., Li, L., Hedrick, J. L. \& Yang, Y. Y. Biodegradable nanostructures with selective lysis of microbial membranes. Nature Chem. 3, 409-414 (2011).

7 Palermo, E. F. \& Kuroda, K. Structural determinants of antimicrobial activity in polymers which mimic host defense peptides. Appl. Microbiol. Biotechnol. 87, 1605-1615 (2010).

8 Tanaka, M., Motomura, T., Kawada, M., Anzai, T., Yuu, K., Shiroya, T., Shimura, K., Onishi, M. \& Akira, M. Blood compatible aspects of poly(2methoxyethylacrylate) (PMEA) relationship between protein adsorption and platelet adhesion on PMEA surface. Biomaterials 21, 1471-1481 (2000).

9 Hatakeyama, T., Tanaka, M. \& Hatakeyama, H. Thermal properties of freezing bound water restrained by polysaccharides. J. Biomater. Sci. 21, 1865-1875 (2010).
10 Tanaka, M., Hayashi, T. \& Morita, S. The roles of water molecules at the biointerface of medical polymers. Polym. J. 45, 701-710 (2013).

11 Lohmeijer, B. G. G., Pratt, R. C., Leibfarth, F., Logan, J. W., Long, D. A., Dove, A. P., Nederberg, F., Choi, J., Wade, C., Waymouth, R. M. \& Hedrick, J. L. Guanidine and amidine organocatalysts for ring-opening polymerization of cyclic esters. Macromolecules 39, 8574-8583 (2006).

12 Tanaka, M. \& Mochizuki, A. Effect of water structure on blood compatibility - thermal analysis of water in poly(meth)acrylate. J. Biomed. Mater. Res. 68A, 684-695 (2004).

13 Hatakeyama, T., Kasuga, H., Tanaka, M. \& Hatakeyama, H. Cold crystallization of poly(ethylene oxide)-water systems. Thermochim. Acta 465, 59-66 (2007).

14 Morita, S., Tanaka, M., Kitagawa, K., Ozaki, Y. \& Coleman, M. Hydration structure of poly(2-methoxyethyl acrylate): comparison with a 2-mothoxyethyl acrylate model monomer. J. Biomater. Sci. 21, 1925-1935 (2010).

15 Morita, S. \& Tanaka, M. Effect of sodium chloride on hydration structures of PMEA and P(MPC- $r$-BMA). Langmuir 30, 10698-10703 (2014).

16 Schomaker, E. \& Challa, G. Complexation of stereoregular poly(methyl methacrylates). 14. The basic structure of the stereocomplex of isotactic and syndiotactic poly(methyl methacrylate). Macromolecules 22, 3337-3341 (1989).

17 Tateishi, Y., Kai, N., Noguchi, H., Uosaki, K., Nagamura, T. \& Tanaka, K. Local conformation of poly(methyl methacrylate) at nitrogen and water interfaces. Polym. Chem. 1, 303-311 (2010).

18 Furuhashi, Y., Sikorski, P., Atkins, E., Iwata, T. \& Doi, Y. Structure and morphology of the aliphatic polyester poly(d-valerolactone) in solution-grown, chain-folded lamellar crystals. J. Polym. Sci. Part B: Polym. Phys. 39, 2622-2634 (2001).

19 Bai, W., Chen, D., Zhang, Z., Li, Q., Zhang, D. \& Xiong, C. Poly(para-dioxanone)/inorganic particle composites as a novel biomaterial. J. Biomed. Mater. Res. Part B: Appl. Biomater. 90B, 945-951 (2009).

20 Hirata, T., Matsuno, H., Tanaka, M. \& Tanaka, K. Surface segregation of poly(2-methoxyethyl acrylate) in a mixture with poly(methyl methacrylate). Phys. Chem. Chem. Phys. 13, 4928-4934 (2011).

Supplementary Information accompanies the paper on Polymer Journal website (http://www.nature.com/pj) 